\title{
Identification of Inpatients with Coronavirus Disease 2019 (COVID-19) at Risk of Clinical Deterioration: A Multicenter, Retrospective Cohort Study
}

\section{Yi Zhang ( $\sim$ czzhangyi1985@126.com )}

China-Japan Friendship Hospital https://orcid.org/0000-0002-4423-1658

\section{Xiaojing Wu}

China-Japan Friendship Hospital

\section{Lan Ni}

Wuhan University Zhongnan Hospital

\section{Lei Chen}

Tongji Hospital of Tongji Medical College of Huazhong University of Science and Technology

Changzhi Zhou

The Central Hospital of Wuhan

\section{Chang Gao}

The First Affiliated Hospital of Soochew University

\section{Jingen Xia}

China-Japan Friendship Hospital

\section{Ye Tian}

China-Japan Friendship Hospital

\section{Sichao Gu}

China-Japan Friendship Hospital

\section{Min Li}

China-Japan Friendship Hospital

\section{Yingying Feng}

China-Japan Friendship Hospital

\section{Jun Duan}

China-Japan Friendship Hospital

\section{Yi Wang}

Tongji Hospital of Tongji Medical College of Huazhong University of Science and Technology Jungang Xie

China-Japan Friendship Hospital

\section{Qiang Guo}

The First Affiliated Hospital of Soochew University 


\section{Jianping Zhao}

Tongji Hospital of Tongji Medical College of Huazhong University of Science and Technology

\section{Yi Hu}

The Central Hospital of Wuhan

\section{Zhenshun Cheng}

Wuhan University Zhongnan Hospital

\section{Qingyuan Zhan}

China-Japan Friendship Hospital

\section{Research}

Keywords: coronavirus disease 2019; clinical deterioration; acute hypoxemic respiratory failure; risk factors

Posted Date: August 11th, 2020

DOI: https://doi.org/10.21203/rs.3.rs-55457/v1

License: (a) (i) This work is licensed under a Creative Commons Attribution 4.0 International License. Read Full License 


\section{Abstract}

Background: Current information is not enough to recognize the risk factors of clinical deterioration and to make medical decisions in COVID-19 patients.

Methods: A retrospective study was performed, with collecting data from medical records of COVID-19 patients in three designated hospitals from January 8, 2020 to May 6, 2020. Clinical data were analyzed between the deteriorated and the non-deteriorated patients, which was defined as either a increase of 2 categories on the modified 6-category ordinal scale, or a decline of $\mathrm{PaO}_{2}$-to- $\mathrm{FIO}_{2}$ ratio more than $100 \mathrm{mmHg}$.

Results: Total 238 patients with COVID-19 were selected, where 31 were deteriorated and 207 were nondeteriorated. In the deterioration group, the case fatality rate was up to $41.9 \%$. Compared with nondeteriorated patients, the deteriorated were older (65.8[IQR 54.3-72.3] vs 54.4[41.0-66.1], $p=0.004$ ) and were more likely to have chronic medical illnesses (17[54.8\%]) vs 92[44.4\%]). Multivariable regression showed that three variables, neutrophil-lymphocyte ratio (NLR) $\geq 3.66(\mathrm{OR}, 9.85 ; 95 \% \mathrm{Cl}, 1.68-57.57)$, hyponatremia (OR, 8.35; 95\% Cl, 1.74-40.16), and presence of ground-glass opacities with consolidation (OR, 5.84; 95\% Cl, 1.24-27.49) were associated with increased odds of clinical deterioration. The variable that inspiring air or traditional oxygen therapy only within 72 hours after admission, indicated a decreased odd of illness progression (OR, $0.075 ; 95 \% \mathrm{Cl}, 0.012-0.465)$.

Conclusions: COVID-19 patients with clinical deterioration had more common extra-pulmonary organ impair in early stage and high case fatality rate. Three factors, NLR $\geq 3.66$, hyponatremia and presence of ground-glass opacities with consolidation were determined as high risk factors in deterioration.

\section{Background}

The outbreak of the novel coronavirus, now known as severe acute respiratory syndrome coronavirus 2 (SARS-CoV-2), has spread globally in the past 4 months [1]. The full spectrum of COVID-19 severity can range from asymptomatic, to symptomatic but mild, to severe (requiring intensive care), even to death [2, 3]. Most COVID-19 patients manifest non-pneumonia or mild pneumonia. However, $14 \%$ of patients' condition is severe and require oxygen therapy, and $5 \%$ is critical with multiple organ dysfunction. Across China, a fatality rate of up to $49 \%$ is observed in critically ill patients [4], which is possibly attributed to inadequate hospital critical care resources. Moreover, an extremely high fatality of critically ill patients is observed in two independent centers in the epicenter $[5,6]$. Identifying which cases need advanced medical services on a priority basis to prevent the illness from progressing is a key problem. However, considering that current information regarding COVID-19 is insufficient, recognizing the risks of clinical deterioration and formulating medical decisions for COVID-19 patients are considered difficult.

A recent study has revealed that older age, high sequential organ failure assessment (SOFA) score, and Ddimer greater than $1 \mu \mathrm{g} / \mathrm{mL}$ are potential risk factors used to identify patients with poor prognosis on admission [6]. However, these factors indicates irreversible pathophysiological processes. Nevertheless, 
several factors, such as body mass index, basic comorbidities, partial pressure of oxygen or $\mathrm{PaO}_{2}$-to- $\mathrm{FIO}_{2}$ ratio (PFR), lymphocyte count, interleukin (IL)-6, and ratio of T cell subsets, are considered beneficial in predicting poor outcomes and deciding on the appropriate management of viral pneumonia [7-9]. Their roles in the identification of COVID-19 patients at risk of clinical deterioration remain unclear. Hence, developing a risk factor-dependent strategy for COVID-19 severity assessment to improve tiered medical services and early warning system is essential.

The current study aimed to improve a prognostic signature-dependent model in at-risk inpatients identified in the early course of COVID-19 and assist medical decisions among patients at risk of clinical deterioration. We believe that our results will facilitate the design and conduct of COVID-19 prevention strategies.

\section{Materials And Methods}

\section{Study Design and Patients}

A multicenter, retrospective, observational study was conducted from January 8, 2020 to May 6, 2020 in three designated hospitals-Zhongnan Hospital of Wuhan University, Tongji Hospital of Tongji Medical College, and Wuhan Central Hospital, Wuhan, China.

We retrospectively studied all adult inpatients ( $\geq 18$ years old) in these three hospitals who had been diagnosed with COVID-19 pneumonia by laboratory confirmation or clinical diagnostic criteria, according to the National Health Commission, China interim guidelines. Patients who died or were discharged between January 10, 2020 and March 20, 2020 were included in our study.

All adult patients underwent real-time reverse-transcription polymerase chain reaction (RT-PCR) testing more than once, to confirm SARS-CoV-2 infection throughout the course of the disease.

A total of 290 hospitalized patients were diagnosed with COVID-19 pneumonia based on typical findings on chest computed tomography (CT) images. A total of 52 patients were excluded: 22 were still in the hospital as of March 20, 9 did not receive SARS-CoV-2 RNA detection, 11 had oxygen therapy with $\mathrm{FiO}_{2}>$ 0.6 upon admission, 2 had unstable hemodynamics upon admission, and 8 had no available key information in their medical records (Fig. 1).

The study was independently approved by the ethics committees of the above mentioned hospitals (2020-21-K16 and TJ-IRB20200418), and all patients provided written informed consent before enrollment.

\section{Data Collection}

Hospitalized patients were identified by reviewing and analyzing the admission logs and histories from all available electronic medical records and patient care resources. For patients who were admitted in the hospital on March 6, 2020, their outcomes were confirmed on March 20, 2020. Once recruited, clinical 
information was recorded systematically each day from admission using a standardized electronic case record form.

\section{Laboratory Procedures}

A total of 192 pneumonia patients received SARS-CoV-2 RNA detection before being admitted to the hospital, 142 of whom had positive results. Throat swab specimens were obtained for RT-PCR reexamination every other day after admission. All 238 inpatients were diagnosed with COVID-19 pneumonia on March 20, 2020.

Routine blood examinations included complete blood count, coagulation profile, and serum biochemical tests within 48 hours after admission. Myocardial enzyme, IL-6, serum ferritin, and procalcitonin levels were also assessed. Chest radiography or CT scan was also performed for all inpatients. The frequency of examinations was determined by the treating physician.

\section{Definitions}

Clinical deterioration was defined as either of the following two criteria from admission to 14 days, whichever came first: (1) an increase of 2 categories on the modified 6-category ordinal scale of clinical status and (2) a decrease of PFR $>100 \mathrm{mmHg}$ at any two independent time points, as confirmed by blood gas analysis. The 6-category ordinal scale comprised the following mutually exclusive categories: category 6 , death; 5 , hospitalization requiring extracorporeal membrane oxygenation (ECMO) and/or invasive mechanical ventilation (IPV); 4, hospitalization requiring high-flow nasal cannula (HFNC) oxygen therapy or non-invasive positive-pressure ventilation (NPPV), but not requiring ECMO and/or invasive mechanical ventilation; 3 , hospitalization requiring traditional supplemental oxygen, including nasal cannula or mask oxygen therapy, but not HFNC or NPPV; 2 , hospitalization not requiring supplemental oxygen; and 1, discharged.

Lymphocytopenia was defined as lymphocyte count $<0.8 \times 10^{9} / \mathrm{L}$, as confirmed by routine blood test. SARS-CoV-2-related acute respiratory distress syndrome (ARDS) was diagnosed according to the Berlin Definition [10] and the WHO guidelines for COVID-19 [11]. Sepsis and septic shock were defined according to the 2016 Third International Consensus Definition for Sepsis and Septic Shock [12]. Acute kidney injury was diagnosed according to the KDIGO clinical practice guidelines [13]. Cardiac injury was diagnosed if the serum concentration of hypersensitive cardiac troponin I (hsTNI) was above the upper limit of the reference range $(>28 \mathrm{pg} / \mathrm{mL})$.

\section{Outcomes}

The primary outcome was 14-day clinical deterioration (the event). The secondary outcomes were time to either hospital discharge or death, length of hospital stay, incidence of SARS-CoV-2-related ARDS, rate of endotracheal intubation and time of weaning off supplemental oxygen, and in-hospital mortality.

The criteria for discharge were as follows: absence of fever for at least 3 days, substantial improvement in both lungs on chest CT, clinical remission of respiratory symptoms, and two throat swab samples 
negative for SARS-CoV-2 RNA obtained at least 24 hours apart.

\section{Statistical Analyses}

Continuous variables are expressed as mean (standard deviation) or median (interquartile range [IQR]), and categorical variables are expressed as number (proportion). Two-group comparisons (nondeterioration vs. deterioration) were conducted by two-sample t-test or Wilcoxon rank-sum test depending on the parametric or non-parametric data for continuous variables and $\chi 2$ test or Fisher's exact test for categorical variables, as appropriate. Differences between the rates of continuing hospital stay, continuing mechanical ventilation, and in-hospital survival were also determined.

We excluded the variables from the univariate analysis if their between-group differences were insignificant, if their accuracy was unconfirmed, and if the number of events was significantly small to calculate the odds ratios (ORs). Considering the total number of deteriorated patients $(n=31)$ and to avoid overfitting in the model, six variables were selected for multivariate analysis based on the previous findings and clinical limitations. Previous studies have shown that older age, lymphopenia, and higher neutrophil-to-lymphocyte ratio (NLR) were associated with the severity of viral pneumonia $[7,8,14]$, which have been commonly observed in critically ill or dead patients with SARS-CoV and Middle East respiratory syndrome (MERS)-CoV infection $[15,16]$. The cutoff value, 3.66, was determined as previously described [17]. Some laboratory findings, including increased LDH level or decreased sodium concentration, showed statistically significant differences between the clinically deteriorated patients, which has not been reported in COVID-19. Ground-glass opacities with consolidation have been proven to present severe lung damage and exudation in SARS-CoV-2 pneumonia [18]. Meanwhile, regarding respiratory physiology, the requirement of higher inspiratory flow and positive pressure mechanical ventilation represents the enhancement of ventilation as hypoxic respiratory failure progresses as a compensatory mechanism. Therefore, we selected older age, NLR, LDH, serum sodium, presence of ground-glass opacities with consolidation in the initial CT scan, and inspired air or traditional oxygen therapy only within 72 hours after admission as the six variables for our multivariate logistic regression model.

A two-sided a value $<0.05$ was considered statistically significant. Statistical analyses were performed using the SAS software version 9.4, unless otherwise indicated.

\section{Results}

\section{Patient Characteristics and Ordinal Scale Outcomes}

On March 20, 2020, 318 adult patients diagnosed with COVID-19 pneumonia were hospitalized in three designated hospitals. Twenty-eight patients were admitted after March 6, 2020, with a time window less than 14 days. 290 inpatients were included initially. A total of 238 inpatients were included in the final analysis, after excluding 52 patients (Fig. 1). The median age was 54.0 (IQR, 42.6-67.3) years, and $42 \%$ were older than 60 years (data not shown). A total of 126 (52.9\%) patients were male. None of the patients had history of exposure to the Huanan Seafood Market. Moreover, 109 (45.8\%) patients had 
chronic diseases. The most common symptoms were fever (86.1\%), cough (72.8\%), and fatigue (43.7\%) (Table 1).

Lymphocytopenia was observed in 95 (39.9\%) patients. The above demographic data and clinical features were similar to those published previously. Furthermore, 192 patients received SARS-CoV-2 RNA detection before admission, with a positive rate of $74.0 \%$ (142 of 192) (Table 2). The remaining 50 patients with negative RNA detection results and 46 patients without RNA detection were admitted according to clinical diagnostic criteria.

The distribution of patients falling into each category of the 6-category scale from admission to day 14 is shown in Fig. 2. Hospitalized patients requiring traditional supplemental oxygen therapy without HFNC, NPPV, or IPV (category 3) accounted for a higher proportion among those hospitalized on the first day, and an increase in more severe outcomes (categories 4-5) was observed within the first 5 days after admission. Additionally, 31 (13.0\%) patients significantly experienced 14-day clinical deterioration. Moreover, 25 patients in the deterioration group experienced an increase of 2 categories, mainly because of the change in respiratory support therapy. Six patients, who initially received positive pressure mechanical ventilation with higher categories, had decreased PFR $>100 \mathrm{mmHg}$ at any two independent time points, as confirmed by blood gas analysis. The other 207 patients clinically improved and were discharged on March 20, 2020.

\section{Clinical Features and Outcomes in Deteriorated Patients}

Deteriorated patients were older (65.8 [IQR, 54.3-72.3] years vs. 54.4 [141.0-66.1], $p=0.004)$ and were more likely to have chronic medical illnesses (17 [54.8\%]) vs. 92 [44.4\%]) than non-deteriorated patients. The incidence rate of hypertension was higher in the deterioration group, with a insignificant difference (12 [38.7\%] vs. 57 [27.5\%], $p=0.201)$ (Table 1).

The deteriorated patients had more severe systemic symptoms than the non-deteriorated, taking the form of fever with maximum temperature $(\operatorname{Tmax}) \geq 38.5^{\circ} \mathrm{C}(21$ [67.7\%] vs. 69 [30.3\%], p<0.001) and myalgia (21 [67.7\%] vs. 73 [35.3\%], $p=0.001$ ). Moreover, dyspnea was more frequently observed in deteriorated patients (18 [58.1\%] vs. 77 [37.2\%], $p=0.027)$. The median duration from symptom onset to hospital admission was not different between the two groups (7.0 [4.0-10.0] days vs. 9.0 [5.0-12.0], $p=0.214$ ) (Table 1).

Only $80.7 \%$ (192/238) patients received SARS-CoV-2 RNA detection before admission. The proportion of false-negative results in SARS-CoV-2 RNA detection was 26\% (50/192). Other 96 patients were diagnosed with COVID-19 based on their clinical diagnostic criteria upon admission. Before admission, the positive rate of SARS-CoV-2 RNA detection was similar in the two group (10 [58.8\%] vs. 132 [75.4\%], $p=0.136$ ) (Table 2).

The baseline lymphocyte count was significantly lower in deteriorated patients than that in nondeteriorated patients $\left(0.7[0.4-0.8] \times 10^{9} /\right.$ L vs $\left.1.0[0.8-1.4], p<0.001\right)$. Levels of aspartate 
aminotransferase and bilirubin were significantly higher in deteriorated patients than those in nondeteriorated patients. Meanwhile, levels of creatine kinase (CK), lactate dehydrogenase (LDH), and myocardial injury biomarkers, including hsTNI, CK isoenzyme MB, and N-terminal pro B-type natriuretic peptide (NT-proBNP), were significantly higher. Levels of serum albumin and sodium serum were lower in deteriorated patients. Meanwhile, levels of ferritin, IL-6, procalcitonin and ESR, considered as key inflammatory signatures throughout the clinical course, were significantly higher in the deterioration group in the early stage of the disease (Table 2).

Although all patients were radiologically diagnosed with COVID-19 through either chest CT or radiography in fever clinics or emergency rooms before admission, only 155 of the 238 hospitalized patients had clear initial chest CT images used for further analysis. Ground-glass opacities were typical findings presented in all 155 initial CT images. It was remarkable that the rate of consolidation observed was evidently higher in the deterioration group (18 [78.3\%] vs. 41 [31.1\%], $p<0.001)$. Reticular lung patterns were observed in $5(21.7 \%)$ in deteriorated, and $6(4.5 \%)$ in non-deteriorated patients, respectively $(p=0.012)$ (Table 3).

80 patients underwent chest CT re-examination 5 days after admission. Follow-up chest CT examination showed an increasing extent and density of lung opacities in all 9 deteriorated patients $(100 \%)$ (Supplementary Table 1).

\section{Other Clinical Outcomes and Treatments}

During hospital stay, respiratory failure $(51.2 \%, 122 / 238)$ was the most common complication. Eight patients were diagnosed with ARDS. The time of weaning off supplemental oxygen and the length of hospital stay were significantly longer in the deterioration group than those in the non-deterioration group (26.0 [24.0-28.0] days vs. 15.0 [7.0-19.0], $p<0.001 ; 26.0$ [26.0, 29.0] days vs. $17.0[11.0,19.0], p<0.001)$. In the deterioration group, the case fatality rate of COVID-19 was $41.9 \%$ (13 of 31). All non-deteriorated patients survived and were discharged (Table 4).

The proportion of receiving antiviral drugs or median time from illness onset to the initiation of antiviral treatment, including oseltamivir or lopinavir-ritonavir, did not differ between the two groups. The proportion of arbidol use was lower in the deterioration group, not only before admission (3 [9.7\%] vs. 58 [28.9\%], $p=0.018$ ) but also within 72 hours after admission (4 [12.9\%] vs. 96 [46.4\%], $p=0.001)$. The proportion of steroid use was higher in the deterioration group (17 [22.6\%] vs. 7 [3.4\%], p< 0.0001$)$ (Table 4).

\section{Risk Factors for Clinical Deterioration}

We included 106 patients with complete data for all variables (75 in the non-deterioration group and 31 in the deterioration group) in the multivariate logistic regression model. We selected older age ( $\geq 65$ years or $<65$ years as a categorical variable), NLR ( $\geq 3.66$ or $<3.66$ ), $\mathrm{LDH}$, serum sodium $(\geq 135 \mathrm{mmol} / \mathrm{L}$ or $<$ $135 \mathrm{mmol} / \mathrm{L}$ ), inspired air or traditional oxygen therapy only within 72 hours after admission (yes or no), and presence of ground-glass opacities with consolidation in initial CT scan (yes or no) as the six 
variables for our multivariate logistic regression model. We found that NLR $\geq 3.66(\mathrm{OR}, 9.85 ; 95 \%$ confidence interval $[\mathrm{Cl}], 1.68-57.57)$, hyponatremia (OR, 8.35; $95 \% \mathrm{Cl}, 1.74-40.16)$, and presence of ground-glass opacities with consolidation in the initial chest CT scan (OR, 5.84; 95\% Cl, 1.24-27.49) were associated with increased odds of clinical deterioration. Inspired air or traditional oxygen therapy only within 72 hours after admission indicated a protective effect from illness progression $(\mathrm{OR}, 0.075 ; 95 \% \mathrm{Cl}$, 0.012-0.465) (Fig. 3).

\section{Discussion}

31 hospitalized patients with confirmed SARS-CoV-2 infection, characterized by progressive hypoxemia and advanced respiratory support requirement, and with deteriorating clinical status were included in this study. It is worth mentioning that these patients had $\mathrm{FiO}_{2}<0.6$, with stable hemodynamics upon admission, meeting our inclusion criteria. Considering their clinical status on admission, physicians were able to designate an inappropriate treatment site for these patients and underestimated the development of their illnesses. Thus, we aimed to identify some easily accessible signatures for early warning and triage before severe respiratory failure and extrapulmonary organ injury are observed.

In our study, oxygenation is still a central element in the assessment of deterioration. The 7-category ordinal scale is able to capture a broad range of clinical status and track the status change of patients from admission based on management of influenza A and COVID-19[19, 20]. Temporary hospitals and inadequate medical teams adversely strain the borders of traditional intensive care units (ICUs), thus, we improved the practical 6-category ordinal scale dependent on the mode of respiratory support, instead of the traditional 7-category scale. If the patients experienced moderate hypoxia (categories 3-4), PFR was also introduced into the criteria.

Lymphopenia was significantly observed in patients with severe COVID-19 [5, 21]. In COVID-19, neutrophil counts were higher in non-survivors than those in survivors [22, 23], similar as in SARS or MERS[15, 16, $24,25]$. Assessment of NLR rather than lymphocyte count was performed when evaluating the patient's immune status, considering the effect of the virus on the immune system and the potential probabilities for co-infection [26]. With NLR assessment, the discrepancy of the baseline was enhanced, facilitating the identification of at-risk patients.

Originally, we revealed that hyponatremia predicted the severity of COVID-19. Hyponatremia has been reported to be typical in severe community-acquired pneumonia considering that Legionella pneumophila is the causative agent for this type of pneumonia [27]. Electrolyte abnormalities caused by syndrome of inappropriate anti-diuretic hormone secretion [28] and renal tubular damage, indicate that some special pathogens possibly complicate Fanconi syndrome-like features [29]. It has been confirmed that SARSCoV-2 uses the same cell entry receptor, angiotensin-converting enzyme 2, which is highly expressed in airway epithelial cells [30] and renal tubular epithelial cells [31]. Although extrapulmonary organ injury caused by SARS-CoV-2 infection will be confirmed based on further studies, hyponatremia should be an early risk factor of illness progression involving the kidney. 
Prehospital chest CT plays a crucial role in the rapid identification and early diagnosis of suspected COVID-19 cases without positive results of RNA detection. In this study, the presence of ground-glass opacities with consolidation, on initial chest CT was associated with COVID-19 deterioration. Of note, follow-up chest CT examination revealing a rapid progression of consolidation in a short duration suggested clinical exacerbation.

In this study, requirement of advanced respiratory support was not observed in the early stage of the disease, implying that inspired air or traditional oxygen therapy only within 72 hours after admission in our study was associated with a low probability of deterioration. MERS showed a deteriorating progress with the median interval from symptom onset to the emergency department visit of 1 day, to ICU admission of 2 days, and to intubation of 4.5 days [32]. In COVID-19, the clinical courses of major outcomes from illness onset were relatively more moderate than those in MERS. The duration from symptom onset to ARDS diagnosis was 8-12 days $[5,6]$, and the duration from ARDS diagnosis to intubation was approximately 14 days [6]. Recognizing hypoxic respiratory failure in a longer time window was considered a limitation. However, inspired air or traditional oxygen therapy only in the early stage represents a better clinical phenotype within 2 weeks. This point could help us to tier medical services and arrange medical resources reasonably.

A recent study has shown that myocardial injury is a common condition among patients hospitalized with COVID-19, and it is associated with a higher risk of in-hospital mortality [33]. Our findings highlight this complication, including new or worsening heart failure or myocardial injury, were common in patients with clinical deterioration, because of the requirement of advanced supplemental oxygen in critically ill inpatients. Lower albumin levels in severely ill patients might be a possible result of capillary leak, which may be due to SARS-CoV-2 infection. Additionally, in deteriorated patients, increased bilirubin, CK and LDH suggested systemic damage of multiple organs, which had been confirmed in autopsies [34, 35].

It has been suggested that inflammatory storms play a key role in the progression of COVID-19 [36]. Elevated ESR, CRP, blood IL-6, and serum ferritin levels might be underestimated in predicting the severity of illness with poor outcomes. Solid evidence to comprehensively understand the association between clinical phenotypes and immune pathogenesis is still required. Additionally, because of insufficient medical resources, a large-scale testing of inflammatory markers should be evaluated when establishing a severity assessment system of COVID-19.

Our study has some limitations. First, because of its retrospective study design, not all laboratory tests were performed in patients within 48 hours, including the assessment of IL- 6 and serum ferritin. Therefore, their role might be underestimated in predicting deterioration. Second, by excluding patients who were still in the hospital on March 20, suggesting that severe COVID-19 patients had longer duration of hospital stay than non-severe patients, the ratio of clinical deterioration was underestimated to some extent. Third, some clinically diagnosed patients, without laboratory confirmation in the hospital, were excluded in our study, resulting in possible bias. Finally, all the four risk factors, which identified COVID-19 inpatients at risk of clinical deterioration, should be further verified in a prospective cohort. 
To the best of our knowledge, this is the first retrospective cohort study to identify inpatients with COVID19 at risk of clinical deterioration. COVID-19 patients with clinical deterioration had more common extrapulmonary organ impairment in the early stage of the disease and higher case fatality rate than that without clinical deterioration. We found that NLR $\geq 3.66$, hyponatremia, and presence of ground-glass opacities with consolidation were considered to be high risk factors for clinical deterioration. Inspired air or traditional oxygen therapy only within 72 hours after admission in our study was associated with a low probability of deterioration.

\section{Declarations}

\section{Ethics approval and consent to participate}

The study was approved by the Ethics Committees of the in three designated hospitals- Zhongnan Hospital of Wuhan University, Tongji Hospital of Tongji Medical College, and Wuhan Central Hospital, Wuhan, China (2020-21-K16 and TJ-IRB20200418). All participants provided written informed consent prior to enrollment. When the patient was unable to provide consent because of consciousness disturbance, the next of kin provided written informed consent.

\section{Consent for publication}

Written informed consent for publication was obtained from all participants. The corresponding author had full access to all the data in the study and had final responsibility for the decision to submit for publication.

\section{Availability of data and material}

The datasets used and/or analysed during the current study are available from the corresponding author on reasonable request.

\section{Competing interests}

The authors declare that they have no competing interests.

\section{Contributors}

QY Zhan, ZS Cheng and Y Zhang had full access to all of the data in the study and take responsibility for the integrity of the data and the accuracy of the data analysis. QY Zhan, ZS Cheng and Y Hu decided to publish the paper. QY Zhan, ZS Cheng, Y Zhang, JP Zhao and Q Guo provided input on the study design. QY Zhan and $Y$ Zhang were responsible for acquisition, analysis, and interpretation of data. $Y Z$ Zhang and XJ Wu drafted the manuscript. QY Zhan, ZS Cheng, Y Zhang, JP Zhao and Q Guo and Y Zhang critically revised the manuscript. XJ Wu, L Ni, L Chen and CZ Zhou contributed to statistical analysis. XJ Wu and SC Gu gave valuable suggestions for data analysis. All authors contributed to conducting the study.

\section{Funding}


This study was supported by Zhejiang University Special Scientific Research Fund for COVID-19 Prevention and Control (No. 2020XGZX008); National Natural Science Foundation of China-Youth Program (No. 81700083); HUST COVID-19 Rapid Response Call (No. 2020kfyXGYJ015); CAMS Innovation Fund for Medical Sciences (No. 2018-12M-1-003) and Non-profit Central Research Institute Fund of CAMS (No. 2019TX320006). Both funding sources have no role in the design of this study and will not have any role during its execution, analyses, interpretation of the data, or decision to submit results.

\section{Acknowledgments}

We would like to express our gratitude to all involved internal medicine residents, pulmonary and critical care fellows, and pulmonary and critical care staff members and nurses in Emergency Rescue Team, China-Japan Friendship Hospital, for their contribution to this project.

\section{References}

[1] WHO. Coronavirus disease (COVID-19) Pandemic. https://www.who.int/emergencies/diseases/novelcoronavirus-2019 (accessed July 14, 2020).

[2] Tian S, Hu N, Lou J, et al. Characteristics of COVID-19 infection in Beijing. J Infect. 2020;80(4):401406.

[3] Guan W, Ni Z, Hu Y, et al. Clinical Characteristics of Coronavirus Disease 2019 in China. N Engl J Med. Published online February 28, 2020. doi: 10.1056/NEJMoa2002032N Engl J Med. 2020; 382:1708-1720

[4] Wu Z, McGoogan JM. Characteristics of and Important Lessons From the Coronavirus Disease 2019 (COVID-19) Outbreak in China: Summary of a Report of 72314 Cases From the Chinese Center for Disease Control and Prevention. JAMA. 2020;323(13):1239-1242

[5] Yang X, Yu Y, Xu J, et al. Clinical course and outcomes of critically ill patients with SARS-CoV-2 pneumonia in Wuhan, China: a single-centered, retrospective, observational study. Lancet Respir Med. 2020;8(5):475-481

[6] Zhou F, Yu T, Du R, et al. Clinical course and risk factors for mortality of adult inpatients with COVID-19 in Wuhan, China: a retrospective cohort study. Lancet. 2020;395(10229):1054-1062

[7] Shi S, Li H, Liu M, et al. Mortality prediction to hospitalized patients with influenza pneumonia: PO(2) /FiO(2) combined lymphocyte count is the answer. Clin Respir J. 2017;11(3):352-360

[8] Viasus D, Pano-Pardo JR, Pachon J, et al. Factors associated with severe disease in hospitalized adults with pandemic (H1N1) 2009 in Spain. Clin. Microbiol. Infect. 2011; 17, 738-746

[9] Guo L, Wei D, Zhang X, et al. Clinical Features Predicting Mortality Risk in Patients With Viral Pneumonia: The MuLBSTA Score. Front Microbiol. 2019;10:2752 
[10] Ranieri VM, Rubenfeld GD, Thompson BT, et al. Acute respiratory distress syndrome: the Berlin Definition. JAMA. 2012; 307: 2526-2533

[11] WHO. Coronavirus disease (COVID-19) technical guidance: Patient management.https://www.who.int/emergencies/diseases/novel-coronavirus-2019/tech nicalguidance/patient-management (accessed July 14, 2020)

[12] Singer M, Deutschman CS, Seymour CW, et al. The Third International Consensus Definitions for Sepsis and Septic Shock (Sepsis-3). JAMA.2016; 315 (8), 801-810

[13] Khwaja A. KDIGO clinical practice guidelines for acute kidney injury. Nephron Clin Pract. 2012; 120: c179-184.

[14] Russell CD, Parajuli A, Gale HJ, et al. The utility of peripheral blood leucocyte ratios as biomarkers in infectious diseases: A systematic review and meta-analysis. J Infect. 2019; 78(5): 339-348

[15] Hui DSC, Zumla A. Severe Acute Respiratory Syndrome: Historical, Epidemiologic, and Clinical Features. Infect Dis Clin North Am. 2019; 33 (4), 869-889

[16] Kim ES, Choe PG, Park WB, et al. Clinical Progression and Cytokine Profiles of Middle East Respiratory Syndrome Coronavirus Infection. Korean Med Sci. 2016; 31 (11), 1717-1725

[17] Forget P, Khalifa C, Defour JP, et al. What is the normal value of the neutrophil-to-lymphocyte ratio? BMC Res Notes. 2017; 10(1):12

[18] Chung M, Bernheim A, Mei X, et al. CT Imaging Features of 2019 Novel Coronavirus (2019-nCoV). Radiology. Published online February 4, 2020. doi: 10.1148/radiol.2020200230

[19] Beigel JH, Tebas P, Elie-Turenne MC, et al; IRC002 Study Team. Immune plasma for the treatment of severe influenza: an open-label, multicentre, phase 2 randomised study. Lancet Respir Med. 2017; 5:500511.

[20] Cao B, Wang Y, Wen D, et al. A Trial of Lopinavir-Ritonavir in Adults Hospitalized with Severe Covid19. N Engl J Med. 2020; 382(19):1787-1799

[21] Huang C, Wang Y, Li X, et al. Clinical features of patients infected with 2019 novel coronavirus in Wuhan, China. Lancet. 2020; 395 (10223):497-506

[22] Wang D, Hu B,Hu C, et al. Clinical Characteristics of 138 Hospitalized Patients With 2019 Novel Coronavirus-Infected Pneumonia in Wuhan, China. JAMA. 2020;323(11):1061-1069

[23] Qin C, Zhou L, Hu Z, et al. Dysregulation of immune response in patients with COVID-19 in Wuhan, China. Clin Infect Dis. Published online March 12, 2020. doi: 10.1093/cid/ciaa248 
[24] Leung GM, Rainer TH, Lau FL, et al; Hospital Authority SARS Collaborative Group. A clinical prediction rule for diagnosing severe acute respiratory syndrome in the emergency department. Ann Intern Med. 2004;141(5):333-342

[25] Alfaraj SH, Al-Tawfiq JA, Assiri AY, et al. Clinical predictors of mortality of Middle East Respiratory Syndrome Coronavirus (MERS-CoV) infection: A cohort study. Travel Med Infect Dis. 2019;29:48-50.

[26] Naess A, Nilssen SS, Mo R, et al. Role of neutrophil to lymphocyte and monocyte to lymphocyte ratios in the diagnosis of bacterial infection in patients with fever. Infection. 2017;45(3):299-307.

[27] Honigsbaum M.Legionnaires' Disease: Revisiting the Puzzle of the Century. Lancet. 2016; 388 (10043), 456-457

[28] Schuetz P, Haubitz S, Christ-Crain M, et al; ProHOSP Study Group. Hyponatremia and Anti-Diuretic Hormone in Legionnaires' Disease. BMC Infect Dis. 2013;13:585

[29] Kinoshita-Katahashi N, Fukasawa H, Ishigaki S, et al. Acquired Fanconi syndrome in patients with Legionella pneumonia. BMC Nephrol. 2013;14:171

[30] Lan J, Ge J, Yu J, et al. Structure of the SARS-CoV-2 spike receptor-binding domain bound to the ACE2 receptor. Nature. 2020;581(7807):215-220

[31] Pan X, Xu D, Zhang H, et al. Identification of a potential mechanism of acute kidney injury during the COVID-19 outbreak: a study based on single-cell transcriptome analysis. Intensive Care Med. 2020;46(6):1114-1116

[32] Arabi YM, Arifi AA, Balkhy HH, et al. Clinical course and outcomes of critically ill patients with Middle East respiratory syndrome coronavirus infection. Ann Intern Med. 2014;160(6):389-397

[33] Shi S, Qin M, Shen B, et al. Association of Cardiac Injury With Mortality in Hospitalized Patients With COVID-19 in Wuhan, China. JAMA Cardiol. Published online March 25, 2020. doi:

10.1001/jamacardio.2020.0950

[34] Xu Z, Shi L, Wang Y, et al. Pathological findings of COVID-19 associated with acute respiratory distress syndrome. Lancet Respir Med. 2020;8(4):420-422.

[35] Yao X, Li T , He Z, et al. A Pathological Report of Three COVID-19 Cases by Minimally Invasive Autopsies. Zhonghua Bing Li Xue Za Zhi. 2020;49(5):411-417.

[36] Chen G, Wu D, Guo W, et al. Clinical and immunological features of severe and moderate coronavirus disease 2019. J Clin Invest. 2020;130(5):2620-2629

\section{Tables}


Due to technical limitations the tables are available as downloads in the Supplemental Files.

\section{Figures}

Figure 1

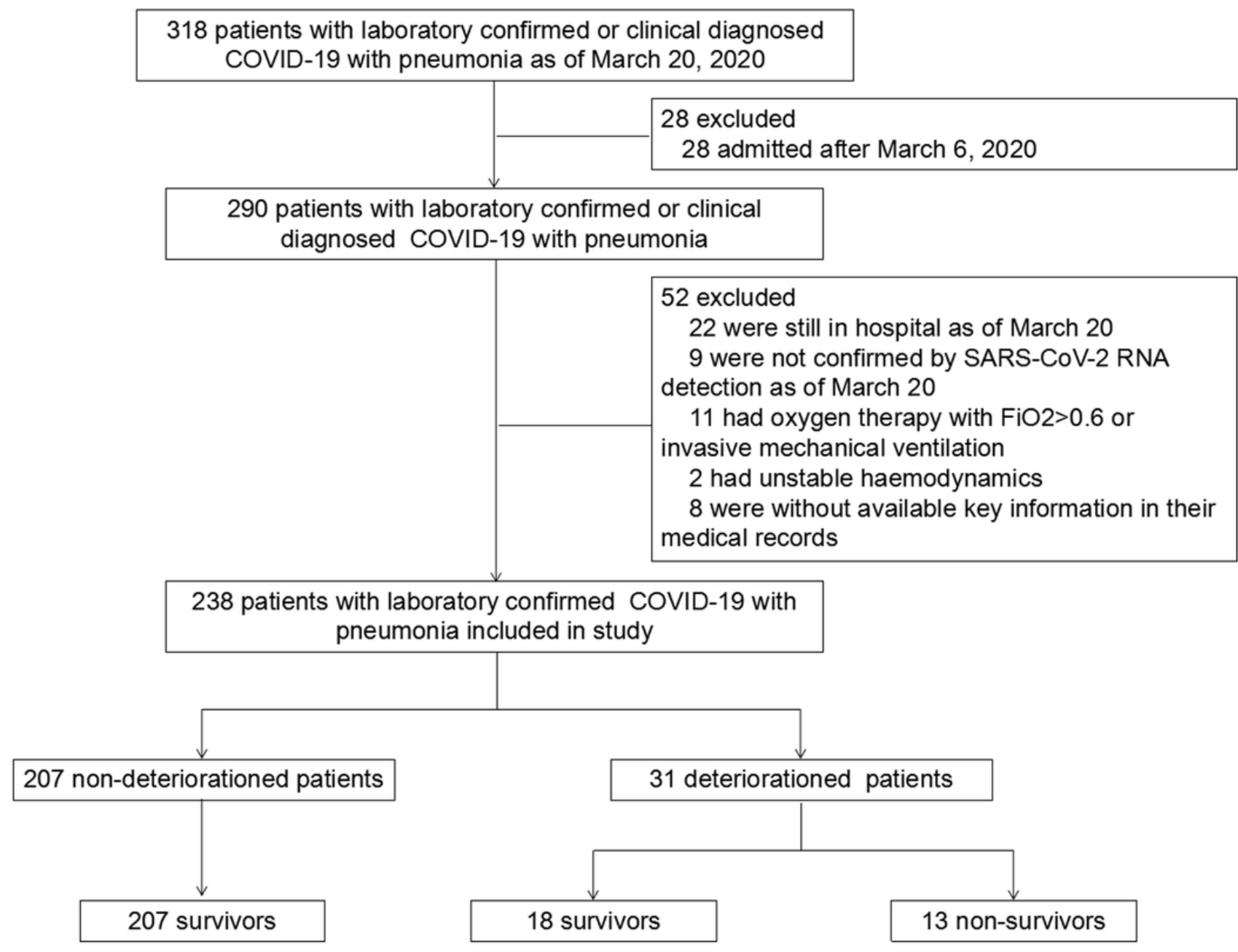

\section{Figure 1}

Study flow diagram. COVID-19, coronavirus disease 2019, SARS-CoV-2, severe acute respiratory syndrome coronavirus 2, $\mathrm{FiO} 2$, fraction of inspired oxygen. 


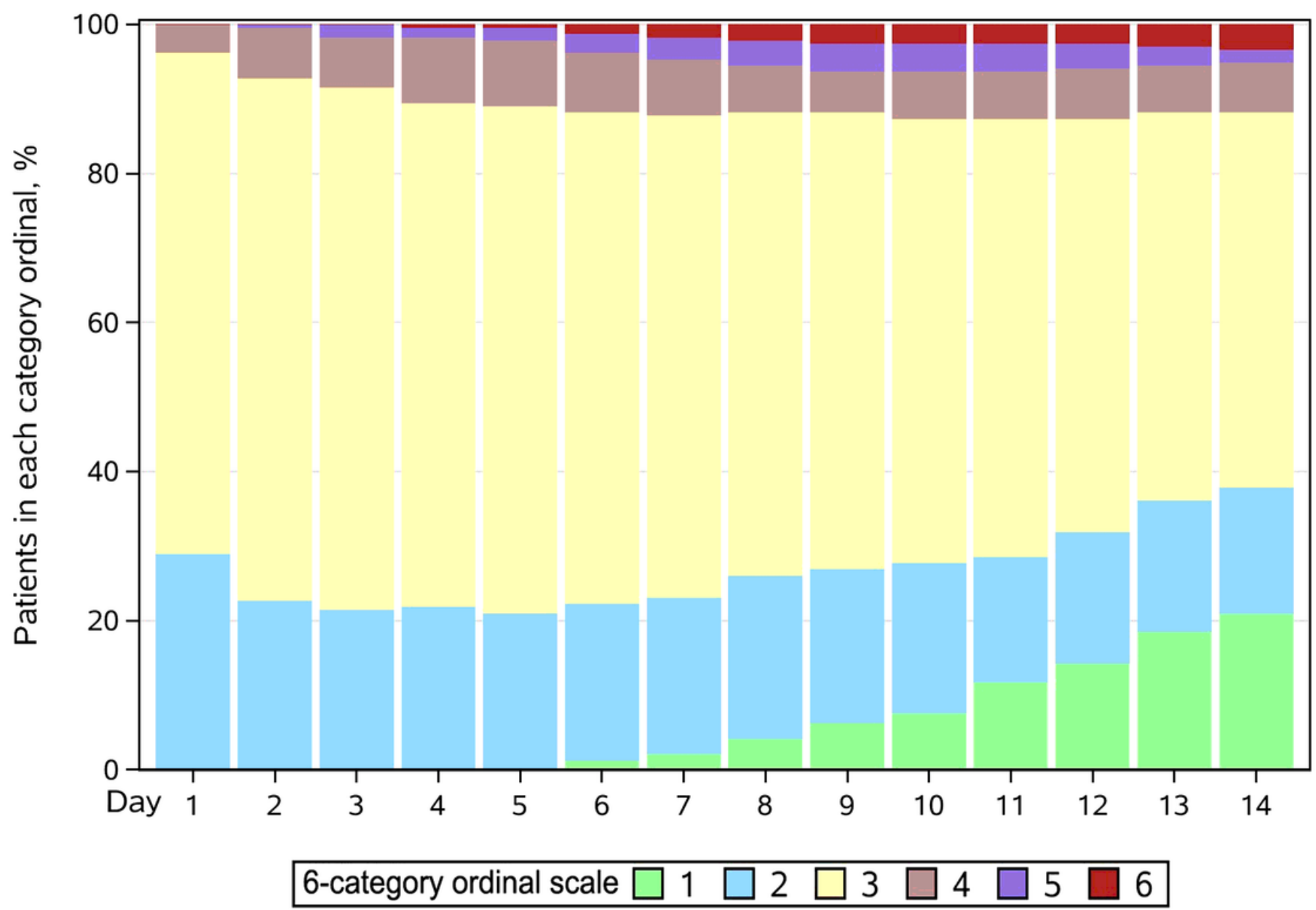

Figure 2

Distribution of proportion falling into each category of the 6-category ordinal scale from admission to day 14. Category 6 , death; 5 , hospitalization, requiring extracorporeal membrane oxygenation (ECMO) and/or invasive mechanical ventilation (IPV); 4, hospitalization, requiring high flow nasal cannula oxygen therapy (HFNC) or non-invasive positive pressure mechanical ventilation (NPPV) , but not requiring ECMO and/or invasive mechanical ventilation; 3 , hospitalization, requiring traditional supplemental oxygen, including nasal cannula or mask oxygen therapy, but not HFNC or NPPV; 2 , hospitalized, not requiring supplemental oxygen; 1 , discharged. 


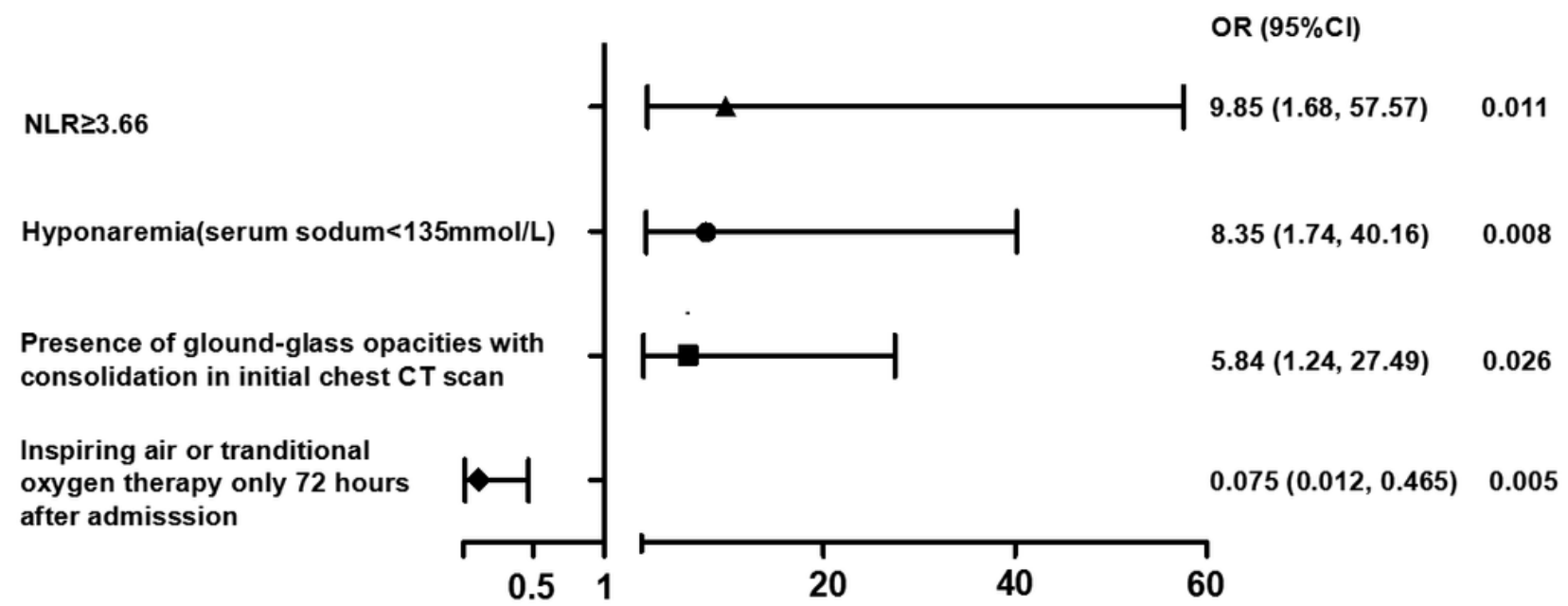

Figure 3

Multivariate logistic regression analysis of risk factors for clinical deterioration in adult inpatients with COVID-19.

\section{Supplementary Files}

This is a list of supplementary files associated with this preprint. Click to download.

- suppltable1.pdf

- suppltable2.pdf

- table1.pdf

- table2.pdf

- table3.pdf

- table4.pdf 